Linguistique, littérature, didactique

\title{
La préparation de l'écriture : vers un concept didactique à forte pertinence
}

\section{Bernadette Kervyn}

\section{(2) OpenEdition}

1 Journals

\section{Édition électronique}

URL : https://journals.openedition.org/pratiques/10259

DOI : 10.4000/pratiques.10259

ISSN : 2425-2042

Éditeur

Centre de recherche sur les médiations (CREM)

Référence électronique

Bernadette Kervyn, «La préparation de l'écriture : vers un concept didactique à forte pertinence », Pratiques [En ligne], 189-190 | 2021, mis en ligne le 09 juillet 2021, consulté le 23 juillet 2021. URL http://journals.openedition.org/pratiques/10259; DOI : https://doi.org/10.4000/pratiques.10259

Ce document a été généré automatiquement le 23 juillet 2021.

(c) Tous droits réservés 


\title{
La préparation de l'écriture : vers un concept didactique à forte pertinence
}

\author{
Bernadette Kervyn
}

\section{La préparation de l'écriture dans l'espace conceptuel du démarrage de l'écriture}

\subsection{Concepts disponibles pour étudier le démarrage de l'écriture}

1 En tant que didacticien, quand on étudie le processus scriptural et plus particulièrement son démarrage, on dispose aujourd'hui de plusieurs concepts qui rendent compte des investigations de ce pan de l'activité scripturale et qui en structurent l'approche. Ces concepts - terme que nous utiliserons pour désigner des notions répondant aux critères définitoires de stabilité, de spécificité, d'intensité ou de puissance de condensation, de pertinence ou d'utilité euristique et praxéologique (Jorro, 2014 ; Mias, 2014 ; Daunay, Reuter \& Schneuwly, 2011 ; Reuter et al., 2013) - sont les suivants : planification (par exemple Fayol, 2013 ; Alamargot \& Chanquoy, 2004 ; Piolat \& Pélissier, 1998), avant-texte (Fenoglio \& Chanquoy, 2007), brouillon (notamment Fabre-Cols, 2004 ; Boré, 2000 ; Recherches, 2011 ; Doquet, 2011; Lumbroso, 2007), écrit(s) intermédiaire(s) ou de travail (Chabanne \& Bucheton, 2000 ; Bucheton \& Chabanne, 2002 ; Chabanne, 2011 ; Bucheton, 2014). Ces concepts ont en commun le fait de traduire et de nourrir un intérêt pour la dimension processuelle et temporelle de l'écriture. De même, ils concernent tous l'écriture en émergence, notamment marquée par des opérations cognitivo-verbales anticipatrices (Kervyn \& Faux, 2014 ; Coulange, Kervyn \& Plissonneau, 2019'1).

2 Le travail épistémologique dont ils sont le fruit ancre la plupart d'entre eux dans une ou dans plusieurs disciplines contributoires pour la didactique $\mathrm{du}$ français (principalement la psychologie, la linguistique et la littérature) ainsi que, de façon plus 
ou moins marquée et spécifique, dans le champ didactique (Kervyn \& Faux, ibid.), où le processus scriptural est étudié de manière particulière, au travers du prisme complexe de son enseignement et de son apprentissage.

3 À la lecture des textes officiels ainsi que des documents ressources sur l'enseignement de l'écriture à destination des professionnels (enseignants et formateurs), telle la conférence de consensus Écrire et rédiger (Brissaud \& Fayol, 2018) ou le dossier thématique sur Les écrits de travail des élèves (Crinon \& Eveleigh, 2018), on constate que ces concepts, à l'exception de celui d'avant-texte, sont largement relayés et utilisés dans la sphère scolaire.

\subsection{La préparation de l'écriture : un terme commun, flou et enchevêtré aux concepts existants}

4 Au niveau professionnel comme en recherche en didactique ou dans des travaux incluant une perspective acquisitionnelle, on rencontre également, à propos de l'écriture, le terme a priori plus commun de préparation. Le plus souvent enchevêtré aux concepts précités, on le trouve sous des formes quelque peu variables : « se préparer à rédiger ", « se préparer à l'écriture », « préparer l'écriture ", « préparer l'écriture avec les élèves ", " préparer les élèves à rédiger ", " phase de préparation ", " préparation à l'écriture ", " préparation de l'écriture ", " préparation à la phase d'écriture ", « activités préparatoires à l'écriture ", " écrit préparatoire ", " dictée préparée ", « la préparation, l'utilisation du brouillon, la production de textes, la révision de textes »...

5 L'addition de ces formes nominales, verbales et adjectivales, loin d'être anecdotiques ou limitées à quelques sources, fait apparaitre qu'il s'agit d'un terme relativement fréquent, transversal et peu stabilisé formellement. Ainsi, le programme de français du cycle 3 suggère, parmi les exemples pour la rédaction d'écrits courts, la "préparation à l'écriture en utilisant des brouillons, des schémas, etc.» (Ministère de l'Éducation nationale [MEN], 2018a, p. 18). Les auteurs de la conférence de consensus Écrire et rédiger, évoquée ci-avant, préconisent eux aussi dans leur synthèse « des activités de préparation à la production écrite, comme le brouillon ». Selon eux, «[1]'entrée en écriture doit faire l'objet d'activités de préparation consistant en l'élaboration d'outils de pré-organisation des thèmes et des termes susceptibles d'être ensuite mis en texte " (op. cit.). Le document ou guide Pour enseigner la lecture et l'écriture au CP stipule que la " phase rédactionnelle particulièrement complexe doit avoir été préparée et anticipée et ne peut se faire simplement en écrivant de manière linéaire » (MEN, 2018b, p. 11). Dans leur travail au croisement de la génétique textuelle et de la didactique, C. Oriol-Boyer (2003) et O. Lumbroso (2008) pointent l'un " une écriture [trop souvent] non-préparée » et l'autre l'importance de mettre en œuvre des « écrits préparatoires ». D. Bucheton et J.-C. Chabanne (2002) mettent en avant la nécessité de dispositifs d'aide « pour préparer et accompagner l'écriture ». P. Bachelé et B. Kervyn (2019) insistent sur les gestes langagiers oraux de l'enseignant pour préparer les essais d'écriture avec les élèves de maternelle.

6 Au-delà de ces quelques exemples qui illustrent la présence sous des formes variables et tout au long de la scolarité de ce que nous proposons de regrouper sous la locution ou le terme ${ }^{2}$ de préparation de l'écriture ${ }^{3}$, il est frappant de constater que, contrairement aux concepts précités, nous sommes ici face à un flou notionnel lié au fait que, quelle que soit sa forme, ce terme n'est généralement pas défini. Même s'il se situe parfois dans 
l'environnement discursif et dans la cartographie conceptuelle que dessinent ces concepts, on ne peut le considérer comme partagé et stabilisé conceptuellement en didactique ou pour la didactique.

7 Néanmoins, interpellée par sa fréquence et sa transversalité, nous souhaitons interroger plus précisément ce qu'il désigne et recouvre puis questionner son intérêt potentiel, plutôt que de nous en tenir à ce constat de flou formel et notionnel. Est-il, par ce qu'il désigne, équivalent ou pour partie équivalent aux concepts pointés ? Dans une perspective évolutive et dynamique des concepts et des champs conceptuels, est-il utile d'en faire ou de vouloir en faire un concept spécifiquement didactique?

Pour répondre à ces questions, nous prendrons appui sur des sources diverses traitant d'une part de de la didactique et de ses fonctions, et d'autre part de l'écriture, de son démarrage et de leur enseignement et apprentissage. Pour ce second volet, nous considèrerons des publications à destination des professionnels (par exemple Brigaudiot, 2000, 2004 ; Ouzoulias, 2004 ; Brissaud \& Cogis, 2011 ; Maillot, 2014 ; Chabrillanges, 2017 ; Herbin, 2019), le travail mené durant plusieurs années dans différents contextes de formation ${ }^{4}$ ainsi que des recherches de didactique et de disciplines contributoires sur les pratiques d'écriture à l'école primaire et secondaire (principalement de la grande section (GS) à la fin du collège). Côté didactique, nous convoquerons notamment le travail exploratoire sur le démarrage de l'écriture et la préparation de celle-ci, réalisé en collaboration avec J. Faux (Faux, 2012 ; Faux \& Kervyn, 2018), P. Bachelé (Bachelé \& Kervyn, 2019), L. Dicharry (2019), L. Coulange et G. Plissonneau (Coulange, Kervyn \& Plissonneau, 2019). Nous nous appuierons aussi sur les premières données issues d'une recherche-action-formation Cardie ${ }^{5}$ intitulée " De l'opération cognitivo-verbale d'anticipation scripturale à l'acte scolaire de préparation de l'écriture ». Démarrée en 2017, cette recherche implique une conseillère pédagogique et des enseignants de la GS au cours moyen 1 (CM1) de la circonscription de Tyrosse-Côte Sud (Landes) et vise la production de ressources sur la préparation de l'écriture.

\subsection{Du flou notionnel à un premier essai de définition}

L'adoption d'un point de vue surplombant croisant des sources différentes permet de comprendre que l'impression de flou mise en exergue ci-avant provient aussi du foisonnement et de la diversité que cette locution de préparation de l'écriture encapsule ou du spectre étendu qu'elle recouvre. En effet, quand on analyse davantage ce que ce terme désigne, on constate que, selon les perspectives et les contextes, la préparation de l'écriture peut concerner des tâches ou des situations d'écriture diverses (copie, dictée, dictée à l'adulte, production d'écrits courts ou longs très variés) en français (commentaire, suite de récit...) ou dans d'autres disciplines (analyse d'un document historique, narration de recherche en mathématiques...). Elle s'étend aux scripteurs allant de la maternelle au lycée (voire après). Elle se fait parfois à l'oral, parfois à l'écrit, parfois à l'oral et à l'écrit. Elle est envisagée tant du côté de l'enseignant, principalement comme un geste professionnel d'étayage ou d'ajustement de la tâche, que du côté des élèves, par exemple capables (ou non) de stratégies ou d'opérations anticipatrices, et souvent elle relève des interactions entre enseignants et élève(s). Ce changement de focale se retrouve dans l'instabilité formelle pointée plus haut et, plus précisément, dans la variation au niveau des actants et des objets : parfois ce sont les 
enseignants qui préparent l'écriture avec leurs élèves ou leurs élèves à l'écriture, parfois ce sont les élèves qui préparent l'écriture ou qui se préparent à l'écriture.

Enfin, elle peut porter sur des dimensions ou des aspects variés de l'activité scripturale et avoir des fonctions multiples qui, ici encore, sont exprimés du point de vue tantôt de l'enseignant, tantôt de l'élève : énoncer la tâche d'écriture; préparer son matériel; présenter/se remémorer le fonctionnement des supports et des outils ainsi que l'ajustement de leur usage à la situation d'écriture; clarifier la situation de communication; expliciter ou faire expliciter les critères de réussite; expliciter ce qu'on fait avant d'écrire le produit visé et l'utilité de le préparer ; mettre en avant des procédures précises («comment on s'y prend pour...»); rassurer; engager dans du connu ; se remémorer la consigne ; préciser ce que celle-ci engage ; mettre son énoncé dans sa tête et dans sa bouche; passer d'une formulation en langue de l'oral à un oral scriptural ; dégager les caractéristiques de l'écrit à produire ; écrire des informations et les ordonner sous forme de liste, de tableau, de carte mentale, de schéma, etc. en vue du texte à venir ; échanger à l'oral sur des contenus ou des idées possibles; rappeler le marquage du pluriel ou fournir des aides orthographiques; pointer ou marquer des difficultés pour y prêter attention au moment de l'écriture; enrichir le lexique lié au thème traité ; ajuster la tâche, le discours ou les outils en fonction des élèves ; situer la tâche d'écriture par rapport aux disciplines et aux attendus disciplinaires; solliciter ou construire des connaissances ou des outils sur le type d'écrit attendu.

11 De cette diversité, nous pouvons tout d'abord déduire que la locution préparation de l'écriture, par ce qu'elle recouvre, présente un caractère particulièrement englobant. L'analyse surplombante des usages considérés fait ensuite apparaitre qu'elle sert à désigner une activité d'anticipation en vue de l'écrit final visé, activité menée au démarrage de l'écriture ou en amont de l'écrit final visé. Cette activité, en cohérence avec la proximité discursive et sémantique entre le terme préparation et les concepts précités, s'inscrit dans une approche temporelle et procédurale de l'écriture, et croise voire décline pour partie les concepts précités. Ainsi, réaliser un brouillon ou un écrit intermédiaire peut correspondre à noter des informations et à les organiser sous forme de liste, de tableau, de schéma ou carte mentale (Kervyn, Faux \& Billon, 2014). L'organisation de contenus présents en mémoire en vue du texte à produire comme la recherche et la récupération d'informations en mémoire à long terme (notamment des connaissances sur le type d'écrit à produire) utiles pour la tâche d'écriture sont des fonctions-clés de la planification mises en avant de façon récurrente (Alamargot \& Chanquoy, 2004 ; Fayol, 2013).

Enfin, dans une perspective didactique, il est essentiel d'ajouter, à ces premiers traits définitoires et à cette première acception, une autre caractéristique, à savoir que, par ses usages, ce terme charrie des formats, des organisations, des préoccupations, des besoins, des habitudes, des contraintes, des outils, des fonctions, etc. liés à l'enseignement et/ou à l'apprentissage (en contexte scolaire dans notre corpus ${ }^{6}$ ). En ce sens, le terme de préparation de l'écriture ne désigne pas l'activité ou le processus même d'anticipation. Il s'agit d'un acte et d'un objet d'enseignement et/ou d'apprentissage qui transforme et décline le processus d'anticipation en tâches, activités (" activités préparatoires »), situations, phases (" phase de préparation »), outils (brouillon, écrits de travail ou intermédiaires), démarche ("se préparer à écrire »). C'est pourquoi le caractère élastique de ce terme peut être analysé comme une réponse au fait que l'activité scripturale est plurielle et complexe mais aussi comme une réponse 
appropriée face à la pluralité et à la variabilité des contextes d'enseignement et d'apprentissage (âge et profils des scripteurs, tâche engagée, choix didactique et pédagogique des enseignants, contexte classe ...).

13 Cette inscription dans des contextes d'enseignement et/ou d'apprentissage amène la formulation de questions essentielles du type: sitôt que la situation d'écriture est complexe pour les élèves, quelles dimensions intervenant dans la réalisation de l'écriture gagnent à être anticipées par un travail scolaire de l'ordre de la préparation de l'écriture ? Comment mener ce travail préparatoire avec les élèves et comment le leur enseigner? Comment constituer la préparation de l'écriture en objet enseigné dont les élèves s'emparent en vue d'une progressive autorégulation de leur activité d'écriture?

En résumé, on est en présence d'un terme à priori commun, transversal et fréquent, flou, englobant et élastique (au niveau de sa forme et de ce qu'il désigne). S'il entretient des liens sémantiques et discursifs étroits avec les concepts de planification et d'avanttexte (issus de travaux de psychologie et de linguistique), avec celui de brouillon (étudié en littérature, en linguistique et en didactique) et avec ceux d'écrits intermédiaires et de travail (plus ancrés en didactique de l'écriture), c'est avant tout au sens où il les relie, les complète ou les décline en phases, activités, tâches, objectifs, nécessité d'enseignement et d'apprentissage. En cela, il semble présenter à ce jour un intérêt pratique et praxéologique plus qu'il ne répond ou correspond à une exigence ou à une clarté épistémique. Si l'on reprend la question posée au départ, il s'agit alors de se demander s'il est pertinent de faire d'un terme à connotation et fonction pratiques et praxéologiques un concept didactique.

\section{Nécessité d'un détour réflexif par la didactique et ses finalités}

Répondre à la question formulée ci-dessus nous engage à réfléchir aux raisons qui justifient l'élaboration et le développement de concepts didactiques, et plus largement au rôle de ceux-ci en didactique du français. Cette question, dans une logique de poupées russes, implique de revenir aussi sur les fonctions assignées à la didactique (du français), pour mieux définir le rôle potentiel visé par une conceptualisation de la préparation de l'écriture.

16 La didactique est régulièrement définie comme une discipline scientifique à même de produire des connaissances sur l'enseignement et l'apprentissage de contenus disciplinaires ou sur des contenus en tant qu'objets d'enseignement et d'apprentissage référés/référables à des matières scolaires (Simard \& al., 2010 ; Reuter \& al, 2013 p. 69). La structuration, la capitalisation et la visibilité de ces connaissances comme de la didactique s'obtiennent entre autres par l'élaboration de concepts à valeur épistémique, qui éclairent et construisent le réel étudié.

17 En plus de cette fonction épistémique de développement de connaissances, la didactique du français, en tant que discipline théorico-pratique (Halté, 1992), poursuit aussi un but praxéologique au sens où elle fait des pratiques la base et la finalité de la recherche (Daunay \& Reuter, 2008, p. 60)7. Selon les chercheurs, cette dimension praxéologique ainsi définie est plus ou moins prépondérante. Appelant à la distinction entre les espaces de recherche, de pratiques, de prescriptions et de recommandations, 
Y. Reuter (2005) évoque un horizon praxéologique lié un engagement indirect. D’autres, sans omettre l'importance de la pluralité des types de recherche, situent davantage la didactique comme une discipline d'intervention (Bronckart, 2001 ; Goigoux, 2017), la considérant parfois sous l'angle d'une technologie et d'une ingénierie à forte visée praxéologique (Bronckart, 2016). Nous en concluons que «[1]a didactique peut être considérée à la fois comme une discipline de recherche et une discipline d'intervention", tel que le suggèrent C. Simard et al. (2010, p.14), et que les interrogations sur les liens entre théories et pratiques, présentes dès l'origine de la didactique (Daunay \& Reuter, 2008), se poursuivent et caractérisent voire fondent cette discipline.

Prolongeons donc les échanges sur leur articulation. Si l'on acte cette double dimension, on peut avancer que le champ de la didactique ne se limite pas à la production de connaissances. Il englobe la production de ressources pour l'action, c'està-dire pour le terrain scolaire et pour la formation (et, en ce qui nous concerne, pour l'enseignement et l'apprentissage de la préparation de l'écriture ainsi que pour la formation). Penser cet élargissement va de pair avec une vision de complémentarité voire d'imbrication (et non pas seulement de mise en tension) des visées épistémologique et praxéologique ou théorique et pratique. Ne serait-ce que pour produire des ressources robustes, répondant à une exigence scientifique et professionnelle (Kervyn, 2019, 2020b), le didacticien prend appui sur des connaissances relatives aux interactions entre les pôles du triangle didactique et analyse le processus de production, générant de la sorte des connaissances didactiques sur le processus engagé ou sur la transformation opérée (transformation du savoir et des ressources par les acteurs engagés et/ou transformation des acteurs).

En cohérence avec cette double visée, nous envisageons les concepts didactiques non seulement comme des espaces de connaissances ou des objets théoriques (Daunay, Reuter \& Schneuwly, 2011, p. 16) mais aussi comme des ressources pour l'action ou des objets ancrés dans la pratique et tournés vers la pratique.

Cette idée de pertinence à la fois scientifique et sociale permet de préciser la question posée en fin de partie précédente : y a-t-il une utilité sociale et une utilité scientifique à constituer la préparation de l'écriture en concept didactique? Quelle plus-value en termes de connaissance et d'action? La préparation de l'écriture, par ce qu'elle recouvre, dessine-t-elle un espace voire un besoin conceptuel didactique théoricopratique, en relation forte avec des questions professionnelles majeures et en lien avec la cartographie conceptuelle que forment la planification, l'avant-texte, le brouillon, les écrits intermédiaires et de travail?

21 Pour y répondre, nous allons focaliser notre propos sur les besoins théorico-pratiques auxquels la préparation de l'écriture est susceptible de répondre. 


\section{Une utilité épistémologique et praxéologique en réponse à différents besoins}

\subsection{Planification et préparation de l'écriture: des modèles du processus rédactionnel à un modèle du processus scriptural et de son enseignement dans une perspective curriculaire et littéracique}

Le premier besoin est lié à un questionnement sur l'adaptation au contexte scolaire du concept de planification issu des modélisations du processus rédactionnel. À l'école ou dans les supports ou ressources à destination des enseignants et des formateurs, la planification concerne presque exclusivement la rédaction de textes, en concordance avec l'approche cognitive de la rédaction qui l'a mise en évidence (Garcia-Debanc, 1986 ; Alamargot \& Chanquoy, 2002 ; Garcia-Debanc \& Fayol, 2002). Pourtant, dans les classes, l'enseignement et l'apprentissage de l'écriture ne se limitent pas à l'unité texte et à de la tâche de rédaction : les élèves apprennent à écrire (c'est-à-dire à copier, à produire seul ou à plusieurs, à écrire sous la dictée, à dicter) des lettres, des mots, des phrases et des textes divers.

Dans une perspective curriculaire et littéracique scolaire, où sont centrales la progression de l'enseignement et de l'apprentissage de l'écriture ainsi que la multiplicité des tâches, des formes, des situations et des fonctions de l'écriture, cette limitation de la planification au texte interpelle. En effet, une part des fonctions assurées par la planification, tels la récupération et le maintien en mémoire d'informations, est susceptible d'outiller les scripteurs novices qui s'essaient par exemple à l'encodage d'un mot ou d'une phrase, en production ou en dictée (Brigaudiot, 2004 ; Brissaud \& Cogis, 2011 ; David \& Morin, 2013).

De surcroit, insérée dans un modèle de l'écriture en tant qu'activité pluricontrainte et potentiellement complexe (Plane, Olive \& Alamargot, 2010; Plane, Alamargot \& Lebrave, 2010 ; Kervyn, 2020c), la planification est définie comme un ensemble de procédures anticipatrices mises en œuvre en réponse à la complexité rédactionnelle ou en réponse à la complexité pour le scripteur de certaines tâches d'écriture textuelle (Bereiter \& Scardamalia, 1987; Alamargot \& Chanquoy, 2004). Cependant, pour des scripteurs novices, la complexité scripturale ne se limite pas à la production textuelle. Dans le cadre d'un travail scolaire avec des apprentis scripteurs qui s'essaient dès la maternelle à l'écriture et sont confrontés à sa complexité (MEN, 2015; Brigaudiot, 2000 ; Chabrillanges, 2017), il est essentiel d'élargir la mise en œuvre de l'activité anticipatrice aux autres unités scripturales et aux autres tâches d'écriture (copie, dictée à l'adulte et dictée notamment), et de l'envisager tant du côté du scripteur que de l'enseignant qui en fait un objet d'étayage (Bachelé \& Kervyn, 2019; Kervyn, 2020a). Il s'agit alors de dépasser le modèle rédactionnel et textuel qui inclut la planification, pour rendre centrale la question suivante : dès qu'il y a complexité pour le scripteur ou les scripteurs (à l'échelle d'une classe ou d'un niveau scolaire par exemple), qu'est-ce qui gagne à être traité de façon anticipée par un travail scolaire de l'ordre de la préparation de l'écriture?

25 La préparation de l'écriture, en tant qu'acte d'enseignement et/ou d'apprentissage, prend ainsi sens dans un élargissement et un ajustement transpositionnels des modèles $\mathrm{du}$ processus rédactionnel à un modèle du processus scriptural, pensé dans une 
perspective curriculaire et littéracique prenant en compte la complexité et la diversité scripturale. Nous proposons de traduire cette extension par des modifications terminologiques significatives dont rend compte le schéma suivant :

Processus scriptural Kervyn, B. (2020c).

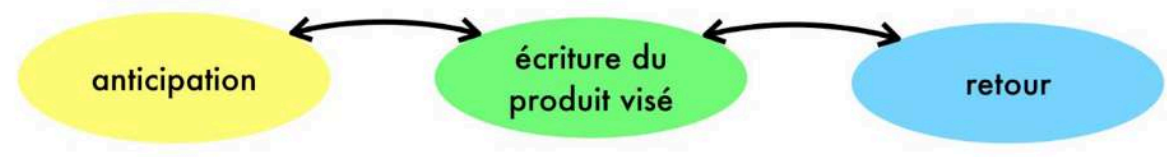

Là où le processus rédactionnel est classiquement décliné en opérations de planification, de textualisation et de révision, le processus scriptural est mis en œuvre au travers d'opérations d'anticipation, d'écriture du produit visé (quel qu'il soit) et de retour ou révision, opérations dont les flèches rendent compte du caractère pour partie enchevêtré. Ces modifications permettent de donner à voir une conception englobante de l'écriture qui ne se réduit pas à la production de textes, qui n'exclut pas la dictée ou la copie, et qui invite à penser de façon transversale l'anticipation scripturale et sa transformation en actes scolaires qu'est la préparation de l'écriture. Dans cette conception, l'anticipation relève d'un modèle scriptural et la préparation de l'écriture prend place dans un modèle d'enseignement de l'écriture. Dans ce modèle scriptural, l'anticipation n'est pas seulement une activité cognitivo-verbale, par exemple de préorganisation des idées de l'écrit à venir. En référence aux nombreux travaux sur la littéracie et dans la lignée de J. Goody (1979; Goody \& Watt, 2006), elle concerne également la matérialité de l'écriture (support, outil, lieu d'écriture) et plus globalement la prise en compte des contraintes et des possibilités liées à la situation d'écriture (objectif, destinataire, exigences, temps imparti, procédures et ressources mobilisables, etc.).

27 C'est parce que la préparation transpose l'anticipation que nous privilégions les termes préparation de l'écriture et préparer l'écriture. Dans une conception processuelle de l'écriture et de son enseignement et apprentissage, la préparation porte bien sur l'écriture du produit visé et non sur l'élève (" préparer l'élève à écrire ", " se préparer à écrire »), même si la transformation en objet scolaire amène légitimement à placer aussi le regard sur l'apprenant. De ce point de vue, nous interprétons la diversité formelle signalée au début de cet article comme une trace de focales plurielles en didactique sur le savoir et sa relation aux pôles élèves et enseignant.

Au travers de ces quelques éléments, il apparait que questionner la préparation de l'écriture, en la rapprochant et distinguant de la planification, et en les mettant en perspective avec des apports didactiques et linguistiques, engage dans une réflexion épistémologique susceptible d'être ressource pour repenser l'enseignement de l'écriture. Au vu du besoin suivant que nous allons exposer, ce pourrait être un enjeu crucial. 


\subsection{Une présence (très) réduite de la préparation de l'écriture dans les pratiques effectives} sur la mise en œuvre du processus scriptural et rédactionnel et sur leur acquisition et, d'autre part, sur les pratiques effectives en matière d'enseignement de l'écriture. Des travaux issus de disciplines différentes et portant sur des scripteurs d'âge contrasté montrent l'importance des opérations d'anticipation. Chez les rédacteurs expérimentés, face à une tâche complexe, jusqu'à $2 / 3$ du temps de l'écriture est consacré à l'anticipation (remémoration d'idées, de contraintes, de stratégies, de la visée ; structuration des idées...) dont dépend fortement la qualité du texte produit (Olive \& Piolat, 2003; Garcia-Debanc \& Fayol, 2002 ; Fayol, 2013). En ce qui concerne les scripteurs novices, au cycle 3 , les meilleurs mettent en place des stratégies d'anticipation quand ils jugent la tâche de production textuelle complexe. Les plus faibles ne le font pas ou de façon inefficace ; ils semblent peu conscients des stratégies possibles ou ne montrent pas de vision procédurale précise (Faux \& Kervyn, 2018). Au niveau du lycée, du collège et de l'école élémentaire, la réalisation individuelle ou collective d'écrits préparatoires, type brouillons instrumentaux ou écrits intermédiaires situés dans une conception processuelle de l'écriture, est susceptible d'aider les élèves à produire des textes de qualité (Lumbroso, 2008 ; Chabanne, 2011 ; Bucheton, 2014; Le Goff \& Larrivé, 2018; Dicharry, 2019; Coulange, Kervyn \& Plissonneau, 2019).

Pour ce qui est du CP, les résultats de la recherche LireÉcrireCP (Goigoux, 2016) ont mis en exergue que les classes où les élèves progressent le plus à l'écrit pratiquent des tâches de préparation de l'écriture (Kervyn, 2018). L'expérimentation Cardie que nous menons avec des classes de la GS au CM1 conforte et étend ces résultats. L'analyse des premières données montre que préparer avec les élèves les tâches d'écriture supposées complexes pour eux et faire de la préparation un objet d'enseignement a un impact majeur sur la mise en œuvre et sur le développement de l'écriture. En outillant les élèves par rapport à la tâche demandée, la préparation facilite l'écriture et la rend plus abordable ; elle améliore la qualité de l'écrit produit (adéquation à la consigne, meilleure prise en compte des normes de l'écrit, écrit plus cohérent, parfois plus long), ce qui allège le travail de correction et de réécriture vite couteux à ce niveau de la scolarité ; elle rend les élèves plus autonomes et plus en réussite.

enéfices visibles engendrent ce que des formateurs et des enseignants lyonnais engagés sur cette thématique nomment "un cercle vertueux »: "comme c'est moins pesant de faire écrire les élèves, je les fais écrire de plus en plus et on progresse tous ». La complexité étant anticipée, les tâches d'écriture se déroulent mieux et mettent davantage les élèves en réussite. Alors qu'auparavant l'écriture était souvent vécue ou perçue, par les élèves et par les enseignants, comme le lieu de difficultés, voire d'échec ou de souffrance, ce déroulement facilité et cette réussite développent chez les premiers une appétence pour l'écriture et chez les seconds l'envie de multiplier les situations d'écriture.

Nous faisons l'hypothèse que ces gains et ce cercle vertueux tiennent à différents facteurs enchevêtrés dont le poids et la nature restent à investiguer. À ce stade exploratoire, les points suivants nous semblent particulièrement importants : figer partiellement le processus scriptural pour consacrer, dans le cadre scolaire, un moment

Pratiques, $189-190 \mid 2021$ 
spécifique à la préparation (sans exclure de la poursuivre durant l'écriture du produit visé) ; faire du travail sur la préparation de l'écriture une activité régulière, ritualisée, au long cours et évolutive ; faire porter le travail sur des tâches d'écriture différentes ; ajuster la préparation aux situations d'écriture, aux élèves et laisser de la place aux styles pédagogiques des enseignants; recourir à l'oral, favoriser les interactions orales et ne pas en faire un moment uniquement écrit, individuel et silencieux ; faire place à la matérialité de l'écriture (supports, outils scripteurs) et au traitement du matériau linguistique (mise en mots, outils orthographiques, connecteurs, formation des lettres...), mobiliser des outils en questionnant leur usage (procédures, matériels, fiches, textes supports...) ; rendre le travail particulièrement explicite (explicitation de la consigne, de la démarche, des critères de réussite, du lien avec le connu, des outils, des procédures, des caractéristiques de la tâche...) ; en faire une activité accompagnée, et un espace d'étayage ; mettre en place une formation et un accompagnement des enseignants portant spécifiquement sur la préparation de l'écriture.

Par contraste, malgré ces travaux convergents ou prometteurs, à l'école, l'écriture reste en général peu (voire très peu) préparée et la préparation de l'écriture n'est quasi pas enseignée, construisant au fil des années chez les élèves la primauté de l'écriture à processus (Oriol-Boyer, 2003) et un démarrage de l'écriture sans réflexion préalable suffisante.

$\mathrm{Au} \mathrm{CP}$, selon les données de la recherche LireÉcrireCP, les enseignants y consacrent en moyenne 5,5 minutes par semaine, toutes tâches d'écriture confondues, $30 \%$ des classes n'en font pas et $30 \%$ entre 1 et 4 minutes par semaine. Dans la mesure où la production de textes est elle aussi très réduite dans les classes constituant l'échantillon de cette recherche mais davantage présente dans les classes réalisant des tâches de préparation de l'écriture, nous nous interrogeons sur la corrélation de ces deux résultats : la mise en œuvre d'un travail de l'ordre de l'anticipation scripturale ne se concentre-t-elle pas sur la production de textes, en conformité avec les recommandations transposant le modèle rédactionnel, alors même que les tâches de copie, de dictée ou d'encodage de mots ou de phrases gagneraient aussi à être préparées?

ce qui concerne la suite de la scolarité, les travaux sur la pratique du brouillon scolaire mettent en avant combien la grande majorité des élèves développent l'habitude de se lancer dans l'écriture d'un brouillon premier jet ou " entrent en textualisation » sans avoir beaucoup anticipé (Boré, 2000 ; Lumbroso, 2010). Par rapport au schéma du processus scriptural proposé précédemment, on est face à une réduction (et parfois une amputation) de l'anticipation par les scripteurs apprenants. En classe, cette réduction va souvent de pair avec une attention forte et un temps long consacré à la réécriture et à la correction. Faire porter une grande partie de la gestion de la complexité scripturale sur la réécriture et la correction ne raccourcit pas systématiquement le temps global de l'écriture et court le risque de les survaloriser et de les rendre très coûteuses pour des scripteurs peu expérimentés.

Pointer ce déséquilibre n'a pas pour objectif de minimiser l'importance des opérations de retour ou de révision (non réductibles d'ailleurs à la réécriture et à la correction) ou la diversité des modes d'écrire (Doquet, 2011) en survalorisant l'écriture à programme. L'enjeu consiste à complexifier la représentation selon laquelle " c'est en écrivant qu'on apprend à écrire » et à faire place à une activité réflexive orale et/ou écrite en amont de l'écriture du produit visé. 

des enseignants, où l'on retrouve ce déséquilibre. Dans les textes officiels et les documents d'accompagnement ressources pour le français (MEN, 2018a, c et d), bien que les élèves soient appelés à mettre en œuvre dès le $\mathrm{CP}$ « une démarche d'écriture de textes : trouver et organiser des idées, élaborer des phrases qui s'enchainent avec cohérence, écrire ces phrases » (MEN, 2018c), la place faite à la révision, à la réécriture, à la correction, à l'évaluation de son texte, à la relecture est largement dominante du cycle 2 au cycle 4 (MEN, 2018a). De même, en formation continue, il est frappant de constater que nombre de questions ou de difficultés formulées par les enseignants ou les formateurs (par exemple, que faire avec les élèves qui oublient ce qu'il faut écrire ou qui ne tiennent pas compte des accords et de la ponctuation quand ils copient ?) sont pensées comme relevant de l'aval de l'écriture du produit visé et ne sont pas envisagées comme pouvant être travaillées en amont.

Nous sommes ici encore face à un rétrécissement du processus scriptural par un modèle d'enseignement de l'écriture qui laisse peu d'espace au travail préparatoire de toutes les tâches d'écriture complexes pour les élèves au fil de la scolarité. Nous pouvons en conclure que le besoin d'une plus grande attention à la préparation de l'écriture se situe tant au niveau de la classe que de la formation et du prescrit. La didactique de l'écriture, comme nous allons le voir dans le troisième et dernier point, n'échappe pas à ce constat.

\subsection{Un investissement didactique encore limité}

Pour terminer cet article, nous voudrions situer notre objet par rapport aux avancées de la didactique de l'écriture. Depuis les années 1980, années parfois considérées en didactique du français comme celles de l'écriture (Barré-De-Miniac, 1996), les recherches se sont poursuivies de façon régulière en bénéficiant d'apports de plusieurs disciplines contributoires (linguistique, sociologie, génétique, psychologie, littérature, pédagogie...). En réponse à la complexité de l'écriture et à la diversité des contextes et des usages, les travaux ont porté sur des dimensions scripturales différentes (dimension linguistique ou affective, supports et outils numériques, situations langagières, geste graphomoteur, dimension cognitivo-verbale...) et sur des niveaux variés (traités avec plus ou moins d'intensité), allant de la maternelle à l'université et la formation tout au long de la vie ${ }^{8}$. Pensés dans leur relation, le pôle enseignant (gestes professionnels, choix didactiques et pédagogiques, efficacité des pratiques, ancrages disciplinaires...) comme le pôle élèves (performances dans des tâches différentes, rapport à l'écriture, culture littéracique, hétérogénéité des publics...) ont fait l'objet de nombreuses recherches ${ }^{9}$.

Si on élargit ce prérédactionnel au préscriptural tel que nous y invite la focalisation sur la préparation de l'écriture considérée dans une perspective curriculaire et littéracique, bien des questions théorico-pratiques restent à traiter ou à approfondir : quelles transformations majeures subit l'anticipation scripturale en devenant préparation de l'écriture ? Quelles sont les caractéristiques et les fonctions stables, inhérentes à la préparation? Sur quelles dimensions portent les variations ? Quelles sont les sources majeures de variation (niveau langagier et scriptural des élèves, tâche, type d'écrit, discipline, choix et profil des enseignants...) ? Quelle place pour l'oral et pour l'écrit, et quelles interactions entre l'oral et l'écrit au sein de la préparation de 
l'écriture ? Quels liens la notion de préparation de l'écriture entretient-elle avec des concepts didactiques ou en circulation en didactique du français (tels qu'explicitation, stratégie, gestes professionnels, interactions langagières, dévolution, erreur, obstacle, rapport à l'écrit, régulation, outil, sujet (élève-apprenant-didactique- scripteur) ou tâche complexe) ? En quoi l'étude de la préparation de l'écriture éclaire-t-elle ces concepts? Que nous apprend-elle sur le démarrage de l'écriture ? Il s'agirait également de poursuivre les recherches sur les effets et les gains, dans des contextes différents, du travail préparatoire de l'écriture, et sur les conditions de leur obtention, ce qui demande de questionner aussi les résistances, les contraintes scolaires (comme l'organisation temporelle et disciplinaire), les inconvénients potentiels ou les passages à risque.

41 Ces questions, issues d'une attention au démarrage de l'écriture et à sa préparation en contexte scolaire, sont programmatiques et leurs traitements prometteurs pour la didactique, en tant que discipline de recherche productrice de connaissances et de ressources robustes, ainsi que pour le terrain de la classe et de la formation. Ces questions programmatiques ajoutées aux différents besoins pointés dans cette quatrième partie suggèrent également que, derrière la fréquence et le flou formel et conceptuel épinglés en début d'article, se cachent une puissance de condensation ainsi qu'une utilité euristique et praxéologique. Grâce à des investigations futures, s'ajouteront, nous l'espérons, à ces deux critères définitoires clés des concepts, ceux de stabilité et de spécificité dans le champ de la didactique de l'écriture.

\section{BIBLIOGRAPHIE}

ALAMARGOT, D. \& CHANQUOY, L. (2002). « Les modèles de rédaction de textes ». In : Fayol, M. (éd.), Production du langage. Paris : Hermès Science Publications, p. 45-65.

ALAMARGOT, D. \& CHANQUOY, L. (2004). « Apprentissage et développement dans l'activité de rédaction de textes ». In : Piolat, A., (dir.), Écriture. Approches en sciences cognitives. Aix-en-Provence : Publication de l'université de Provence, p. 125-146.

BACHELÉ, P. \& KERVYN, B. (2019). « Le rôle des gestes langagiers du maitre dans l'enseignement de l'écriture tâtonnée en Grande section de Maternelle ». In : Kervyn, B., Dreyfus, M. \& Brissaud, C. (dirs), L'écriture dès le début de l'école primaire. Pratiques enseignantes et performances des élèves. Bordeaux : Presses universitaires de Bordeaux, p. 65-84.

BARRÉ-DE-MINIAC, C. (1996). Vers une didactique de l'écriture. Pour une approche pluridisciplinaire. Bruxelles : De Boeck.

BEREITER, C. \& SCARDAMALIA, M. (1987). The psychology of Written Composition. Hillsdale : Lawrence Erlbaum Associates.

BORÉ, C. (2000). « Le brouillon, introuvable objet d'étude ?». Pratiques 105-106, p. 23-49. En ligne : https://doi.org/10.3406/prati.2000.2401.

BRIGAUDIOT, M. (2000). Apprentissage progressif de l'écrit à l'école maternelle. Paris : Hachette. 
BRIGAUDIOT, M. (2004). Première maitrise de l'écrit. CP CE1 et secteur spécialisé. Paris : Hachette.

BRISSAUD, C. \& COGIS. D. (2011). Comment enseigner l'orthographe aujourd'hui ? Paris : Hatier.

BRISSAUD, C. \& FAYOL, M. (dirs) (2018). Écrire et rédiger. Rapport scientifique. Étude de la langue et production d'écrits. Cnesco. En ligne : http://www.cnesco.fr/wp-content/uploads/2018/04/VDEF-rapportBrissaud-Fayol.pdf.

BRONCKART, J.-P. (2001). « La psychologie ne peut être que sociale et la didactique est l'une de ses disciplines majeures ». In : Bernié, J.-P. (dir.), Apprentissage, développement et significations. Bordeaux : Presses universitaires de Bordeaux, p. 19-41.

BRONCKART, J.-P. (2016). Pourquoi et comment devenir didacticien? Villeneuve-d'Ascq : Presses universitaires du Septentrion.

BUCHETON, D. (2014). Refonder l'enseignement de l'écriture. Paris : Retz.

BUCHETON, D. \& CHABANNE, J.-C. (2002). Écrire en ZEP : un autre regard sur les difficultés des élèves. Versailles, CRDP / Paris : Delagrave.

CHABANNE, J.-C. (2011). « Les écrits "intermédiaires" au-delà du brouillon ». Recherches 55, p. 7-20. En ligne : http://www.revue-recherches.fr/wp-content/uploads/2014/06/007-020-Chabanne.pdf. CHABANNE, J.-C. \& BUCHETON, D. (2000). « Les écrits intermédiaires ». Lettre de la DFLM 26, p. 23-27. En ligne : https://doi.org/10.3406/airdf.2000.1424.

CHABRILLANGES, A. (2017). Réussir en production d'écrits (GC-CP puis CE1). Paris : Retz.

COULANGE, L., KERVYN, B. \& PLISSONNEAU, G. (2019). « Préparation de l'écriture à la fin du cycle 4. Quelle actualisation dans des disciplines scolaires? ». In : Dias-Chiaruttini, A. \& Lebrun, M. (dirs), La question de la relation entre les disciplines scolaires : le cas de l'enseignement du français. Namur : Presses universitaires de Namur, p. 75-97.

CRINON, J. \& EVELEIGH, H. (coord.) (2018). « Les écrits de travail des élèves ». Les cahiers pédagogiques 544. DAUNAY, B. \& REUTER, Y. (2008). « La didactique du français : évolution, méthodes et enjeux ». Pratiques 137-138, p. 57-78. En ligne : https://journals.openedition.org/pratiques/1152.

DAUNAY, B. REUTER, Y. \& SCHNEUWLY, B. (dirs) (2011). Les concepts et les méthodes en didactique du français. Namur : Presses universitaires de Namur, collection AIRDF.

DAVID, J. \& MORIN, M.-F. (dirs) (2013). « Premières pratiques d'écriture : état des recherches francophones ». Repères 47. En ligne : https://doi.org/10.4000/reperes.515.

DICHARRY, L. (2019). Enseigner la préparation de l'écriture à des élèves de CM2 en Réseau d'Éducation Prioritaire. Mémoire de master 2 de recherche en didactique du français. Université de Bordeaux. DOQUET, C. (2011). L'Écriture débutante. Pratiques scripturales à l'école élémentaire. Rennes : Presses universitaires de Rennes.

DOQUET, C. \& DAVID, J. (dirs) (2017). «Collecter, interpréter, enseigner l'écriture ». Repères 57. En ligne : https://doi.org/10.4000/reperes.1424.

DOQUET, C. \& DAVID, J. (2018). Collecter, interpréter, enseigner l'écriture. 57. En ligne : https:// journals.openedition.org/reperes/1424.

DUMORTIER, J.-L. (2007). « Recherche en didactique : deux questions et des ébauches de réponses qui suscitent bien d'autres questions ». La Lettre de l'AIRDF 41, p. 21-24. En ligne : https://doi.org/ 10.3406/airdf.2007.1750. 
FABRE-COLS, C. (2004). « Brouillons scolaires et critique génétique : nouveaux regards, nouveaux égards ? ». Linx 51, p. 13-24. En ligne : https://doi.org/10.4000/linx.160.

FAUX, J. (2012). La carte mentale peut-elle permettre aux élèves d'entrer en écriture ? La carte mentale peutelle être révélatrice des profils de scripteur. Mémoire de master 2 de recherche en didactique du français. Université de Bordeaux.

FAUX, J. \& KERVYN, B. (2018). « Entrée en écriture et conduites stratégiques ». In : Brissaud, C., Dreyfus, M. \& Kervyn, B. (dirs), Repenser l'écriture et son évaluation au primaire et au secondaire. Namur : Presses universitaires de Namur, p. 87-119.

FÉNOGLIO, I. \& CHANQUOY, L. (2007). « La notion d'“avant-texte” : point de rencontre pour une compréhension de l'écriture en acte ». Langue française 155, p. 3-7. En ligne : https://doi.org/ 10.3917/lf.155.0003.

GARCIA-DEBANC, C. (1986). «Intérêts des modèles du processus rédactionnel pour une pédagogie de l'écriture ». Pratiques 49, p. 23-49. En ligne : https://doi.org/10.3406/prati.1986.2449.

GARCIA-DEBANC, C. \& FAYOL, M. (2002). « Apports et limites des modèles du processus rédactionnel pour la didactique de la production écrite. Dialogue entre psycholinguistique et didacticien ». Pratiques 115-116, p. 37-50. En ligne : https://doi.org/10.3406/prati.2002.1967.

GoIGOUX, R. (2016). Étude de l'influence des pratiques d'enseignement de la lecture et de l'écriture sur la qualité des premiers apprentissages. Rapport de recherche. En ligne : http://ife.ens-lyon.fr/ife/ recherche/lire-ecrire/rapport/rapport-lire-et-ecrire.

GoIGoUX, R. (2017). « Associer chercheurs et praticiens à la conception d'outils didactiques ou de dispositifs innovants pour améliorer l'enseignement ». Éducation \& didactique 11 (3), p.135-142. En ligne : https://www.cairn.info/revue-education-et-didactique-2017-3-page-135.htm.

GOODY, J. (1979) [1977]. La raison graphique. Paris : Éd. de Minuit.

GoODY, J. \& WATT, I. (2006) [1968]. « Les conséquences de la littératie ». Pratiques 131-132, p. 31-68. En ligne : https://doi.org/10.3406/prati.2006.2115

HERBIN, V. (2019). L'écriture créative au collège. Le site du café pédagogique. En ligne : http:// www.cafepedagogique.net/lexpresso/Pages/2019/01/14012019Article636830473085435258.aspx.

JORRO, A. (2014). «Quels concepts scientifiques dans le champ de la professionnalisation ». In : Jorro, A. (dir.), Dictionnaire des concepts de la professionnalisation. Louvain-la-Neuve : De Boeck, p. 7-13.

KERVYN, B. (2018). Écrire : un acte complexe qui ne se résume pas à la production de textes. Centre Alain Savary, Institut Français de l'Éducation (ENS-Lyon). En ligne : http://centre-alain-savary.enslyon.fr/CAS/education-prioritaire/ressources/theme-1-perspectives-pedagogiques-eteducatives/lire-ecrire-parler-pour-apprendre-dans-toutes-les-disciplines/dossier-lire-ecrire/ copy_of_ecriture-se-construire-une-culture-commune?searchterm=ecriture.

KERVYN, B. (2019). « De la recherche LireÉcrireCP à la production de ressources pour la formation ». Lettre de l'AIRDF 65, 47-50.

KERVYN, B. (2020a). « Rôle des interactions langagières dans la préparation collective de l'encodage de phrases en classe de CP ». Recherches en Éducation 40. En ligne : http://

journals.openedition.org/ree/451. DOI : https://doi.org/10.4000/ree.45

KERVYN, B. (2020b). « De l'utilité de la recherche collaborative pour produire des ressources de formation robustes ». Cahiers de l'Acedle 17 (2). En ligne : http://journals.openedition.org/rdlc/ 7339. DOI : https://doi.org/10.4000/rdlc.7339. 
KERVYN, B. (2020c). Former à l'enseignement de l'écriture, Centre Alain Savary, Institut Français de l'Éducation (ENS-Lyon). Ressources d'interface sur le processus scriptural et son enseignement et modules de formations. En ligne : http://centre-alain-savary.ens-lyon.fr/CAS/documents/ documents-frederique-j/resultats-de-la-recherche-lire-ecrire/former-a-lecriture.

KERVYN, B., DREYFUS, M. \& BRISSAUD, C. (2019). L'écriture dès le début de l'école primaire. Bordeaux : Presses universitaires de Bordeaux.

KERVYN, B. \& FAUX, J. (2014). « Avant-texte, planification, révision, brouillon, réécriture : quel espace didactique notionnel pour l'entrée en écriture ?». Pratiques 161-162. En ligne : https://doi.org/ 10.4000/pratiques.2172.

KERVYN, B., FAUX, J. \& BILLON, V. (2014). « Se servir de la carte mentale pour entrer dans l'écriture. Retour sur un processus d'outillage ». Recherches 60, p. 25-48. En ligne : http://www.revuerecherches.fr/wp-content/uploads/2016/07/025-047_R60_KervynFauxBillon.pdf.

LE GOFF, F. \& LARRIVÉ, V. (2018). Le temps de l'écriture. Grenoble : UGA éditions.

LUMBRoso, O. (2007). « Esquisse d'un dialogue entre didactique de l'écrit et critique génétique : l'élève "auteur-dessinateur"». Revue française de pédagogie 159 (2), p. 119-137. En ligne : https:// www.cairn.info/revue-francaise-de-pedagogie-2007-2-page-119.htm.

LUMBRoso, O. (2008). « Chronique “didactique”. La critique génétique au lycée : auto-consignes et écrits préparatoires ». Le français aujourd'hui 160, p. 119-130. En ligne : https://doi.org/10.3917/ lfa.160.0119.

LUMBroso, O. (2010). « Pour une didactique du prérédactionnel ». Genesis 30, p. 177-184. En ligne : https://doi.org/10.4000/genesis.135.

MAILLOT, P. (2014). Rédiger en orthographiant. Canopé- CRDP Académie de Lille.

MINISTÈRE DE L'ÉDUCATION NATIONALE (2015). Programme d'enseignement de l'école maternelle. Bulletin spécial du 26 mars 2015. En ligne : https://www.education.gouv.fr/au-bo-special-du-26mars-2015-programme-d-enseignement-de-l-ecole-maternelle-3413.

MINISTÈRE DE L'ÉDUCATION NATIONALE (2018a). Programme du cycle 2, 3 et 4. Bulletin officiel de l'Education nationale, $\mathrm{n}^{\circ} 30 \mathrm{du}$ 26-07-2018.

MINISTÈRE DE L'ÉDUCATION NATIONALE (2018b). Pour enseigner la lecture et l'écriture au CP. Un guide fondé sur l'état de la recherche. En ligne : https://eduscol.education.fr/cid129436/pour-enseigner-lalecture-et-l-ecriture-au-cp.html.

MINISTÈRE DE L'ÉDUCATION NATIONALE (2018c). Attendus de fin d'année et repères annuels de progression du CP à la $3^{e}$. En ligne : https://eduscol.education.fr/pid38211/attendus-et-reperes.html.

MINISTÈRE DE L'ÉDUCATION NATIONALE (2018d). Ressources d'accompagnement du programme de français. En ligne : https://eduscol.education.fr/pid34186/francais.html.

MIAS, C. (2014). « Partager des références sur la professionnalisation ». In : Jorro, A. (dir.), Dictionnaire des concepts de la professionnalisation. Louvain-la-Neuve : De Boeck, p. 351-356.

OLIVE, T. \& PIOLAT, A. (2003). « Activation des processus rédactionnels et qualité des textes ». Le Langage et l'Homme 38 (2), p. 191-206.

ORIOL-BOYER, C. (2003). Critique génétique et didactique de la réécriture. Travailler avec les brouillons d'écrivains. Paris : Bertrand-Lacoste.

OUZOULIAS, A. (2004). Favoriser la réussite en lecture : les MACLÉ. Scéren CRDP Académie de Versailles, Paris : Retz. 
PIOLAT, A., \& PÉLISSIER, A. (1998) (éds). La rédaction de textes. Approche cognitive. Lausanne : Delachaux \& Niestlé.

PLANE, S., ALAMARGOT, D. \& LEBRAVE, J.-L. (2010). « Temporalité de l'écriture et rôle du texte produit dans l'activité rédactionnelle ». Langages 177, p. 7-28. En ligne : https://doi.org/10.3917/lang. 177.0007.

PLANE, S., OLIVE, T. \& ALAMARGOT, T. (éds.) (2010). « Traitement des contraintes de la production d'écrit : aspects linguistiques et psycholinguistiques ». Langage 177. En ligne : https://www.cairn.info/ revue-langages-2010-1.htm.

RECHERCHES (2011). Brouillons. 55. En ligne : https://www.revue-recherches.fr/?p=3001.

REUTER, Y. (1996). Enseigner et apprendre à écrire. Paris : ESF.

REUTER, Y. (2005). « Didactique du français : éléments de réflexion et de proposition ». In : Chiss, J.-

L., Davis, J. \& Reuter, Y. (dirs). Didactique du français. Fondements d'une discipline. Bruxelles : De

Boeck, p. 211-231.

REUTER, Y., COHEN-AZRIA, C., DAUNAY, B., DELCAMBRE, I. \& LAHANIER-REUTER, D. (dir.). (2013) [2007]. Dictionnaire des concepts fondamentaux des didactiques. Bruxelles : De Boeck.

SIMARD, C., DUFAYS, J.-L., DOLZ, J. \& GARCIA-DEBANC, C. (2010). Didactique du français langue Première.

Bruxelles : De Boeck Supérieur.

\section{NOTES}

1. Une présentation comparée de ces quatre concepts a été réalisée dans ces publications. Nous ne développerons donc pas ce point dans cet article.

2. Terme est à entendre au sens de locution ou d'expression, raison pour laquelle nous le laissons au singulier.

3. Nous reviendrions sur ce choix dans la partie 4.2. de cet article.

4. Pour la formation, nous prenons principalement appui sur le travail effectué dans 3 contextes professionnels : la formation de formateurs sur la préparation de l'écriture, réalisée à la demande de $\mathrm{P}$. Luyat et L. Roche durant l'année scolaire 2018-2019 pour le groupe Maitrise de la langue de l'Académie de Lyon ; la coordination de la production de ressources sur l'enseignement de l'écriture menée en collaboration avec le Centre Alain Savary et 5 circonscriptions d'Aquitaine entre 2017 et 2020 (Kervyn, 2019, 2020c) ; une formation sur l'écriture au collège à destination du groupe de formateurs Maitrise de la langue de Gironde (formation réalisée en 2017 avec G. Plissonneau et L. Coulange).

5. Conseil académique recherche, développement, innovation, expérimentation ; recherche co-dirigée avec $P$. Bachelé.

6. Ce qui ne signifie pas que la préparation de l'écriture se limite à la sphère scolaire.

7. Cette acception du terme praxéologique se distingue de celle où cet adjectif signifie "qui produit des théories sur la praxis ", que rappelle J.-L. Dumortier (2007).

8. Voir à ce propos le colloque L'enseignement et l'apprentissage de l'écriture de la maternelle à l'université et en formation tout au long de la vie, qui a eu lieu à Bordeaux en 2016.

9. Pour une synthèse concernant l'école primaire, voir l'introduction de l'ouvrage L'écriture dès le début de l'école primaire (Kervyn, Dreyfus \& Brissaud, 2019). 


\section{RÉSUMÉS}

Quand il s'agit de considérer le démarrage de l'écriture, outre les concepts de planification, d'avant-texte, de brouillon et d'écrits intermédiaires ou de travail, on trouve régulièrement, enchevêtré aux précédents, le terme a priori plus commun de préparation (de l'écriture) décliné sous des formes différentes (écrits préparatoires, se préparer à l'écriture, phase préparatoire...). Plutôt que de s'en tenir à un premier constat de flou formel et conceptuel lié à la transversalité de ce terme, cet article analyse ce qu'il recouvre et la pertinence qu'il peut avoir en didactique de l'écriture, dans une perspective curriculaire et littéracique. De cette analyse surplombante émergent différents besoins théorico-pratiques auxquels il répond ainsi que des questions programmatiques pour la didactique de l'écriture. Ces besoins et ces questions révèlent la puissance de condensation de cette notion ainsi que son utilité euristique et praxéologique.

When it comes to considering the start of writing, in addition to the concepts of planning, pretext, draft and intermediate or working writings, we regularly find, intertwined with the previous ones, the a priori more common term of preparation (of writing) declined in different forms (preparatory writings, getting ready to write, preparatory phase...).Rather than confining itself to an initial observation of formal and conceptual vagueness linked to the transversality of this term, this article analyses what it covers and the relevance it may have in the didactics of writing, from a curricular and literary perspective. From this overarching analysis emerge different theoretical-practical needs to which it responds as well as programmatic questions for the didactics of writing. These needs and questions reveal the condensing power of this notion as well as its heuristic and praxeological usefulness.

INDEX

Keywords : didactics of writing, scriptural process, preparation of writing, planning, writing, draft, anticipation, school literacy

Mots-clés : didactique de l'écriture, processus scriptural, préparation de l'écriture, planification, rédaction, brouillon, anticipation, littéracie scolaire

\section{AUTEUR}

\section{BERNADETTE KERVYN}

Université de Bordeaux, Lab-E3D, F-33000 Bordeaux, France 